\title{
Detection of the Spermicide Nonoxynol-9 Via GC-MS
}

\author{
Rabi A. Musah, Angela L. Vuong, Colin Henck, Jason R. E. Shepard \\ Department of Chemistry, University at Albany, SUNY, Albany, NY 12222, USA
}

\begin{abstract}
The spermicide nonoxynol-9 is actually a complex mixture of dozens of closely related amphiphilic compounds, and the chemical properties of this assortment significantly hamper its characterization by GC-MS. The inability to perform routine GC-MS testing on nonoxynol-9 has limited its evidentiary value in forensic casework, which relies heavily on this technique for analysis. A disturbing trend in sexual assault is the use of condoms by assailants, to avoid leaving behind DNA evidence that can connect a perpetrator to a victim. This observation necessitates the development of alternative methods for the analysis of trace evidence that can show causal links between a victim and a suspect. Detection of lubricants associated with sexual assault is one such way to establish this connection. The development of GC-MS methods that permit facile detection of both nonoxynol-9 alone and nonoxynol-9 extracted from other complex matrices that have potential as trace evidence in sexual assault is reported. A detection limit of $2.14 \mu \mathrm{g}$ of nonoxynol-9 is demonstrated, and a detailed mass spectral profile that elaborates on what is known of its structure is provided.
\end{abstract}

Keywords: GC-MS, Forensic, Nonoxynol-9

\section{Introduction}

$\mathrm{D}$ etergents have both hydrophilic and hydrophobic structural features, which make them incompatible with gas chromatography/mass spectrometry (GC-MS). These amphiphilic substances, including nonylphenols and their ethoxylate derivatives (NPEs), have many industrial applications, including use in cosmetics, coatings, and surfactants. One particular NPE, nonoxynol-9, is a spermicidal agent used in the United States as a common component of condom lubricants and other contraceptive products. Condom use has been documented in sexual assault as a means by which criminals can avoid leaving behind incriminating DNA evidence [1-8]. However, in the absence of DNA evidence,

Electronic supplementary material The online version of this article (doi:10.1007/s13361-012-0353-7) contains supplementary material, which is available to authorized users.

Correspondence to: Jason R. E. Shepard; e-mail: jshepard@albany.edu detection of the various components of condom lubricants can serve as a causal link between a victim and suspect [1-4]. In this context, the detection of specific lubricant components has served as a probative link that provides an avenue to corroborate other evidence or information related to the crime. Spermicides in particular have been identified as potential trace evidence in sexual assault, with numerous case studies detailing its contextual value $[2,5,6,8]$. However, detection of nonoxynol-9 for evidentiary purposes has not been fully exploited because its physical characteristics are generally incompatible with the routine equipment most often found in crime labs, GC-MS. The difficulties associated with nonoxynol-9 analysis are imposed mainly by two characteristics: (1) it is non-volatile, which makes its analysis by GC problematic, and (2) it is not a single compound, but rather a complex mixture of highly similar substances of varying molecular weights. Accordingly, detection of lubricant formulations has been performed using microscopy, infrared analysis, and mass spectrometry techniques other than GC-MS [3-5, 9]. 
Significant effort has gone into developing advanced MS techniques for analysis of lubricants, in particular electrospray ionization-MS along with liquid chromatographyMS, matrix-assisted laser desorption ionization (MALDI)MS, and other desorption techniques [4, 6, 10-13]. However, these advanced methods and instrumentation are not widely utilized in crime laboratories. Research on advanced techniques is important, but most crime labs do not have the resources to acquire such instrumentation. While the outsourcing by crime labs of MS testing can serve as an avenue by which to conduct advanced sophisticated MS analyses, performing such testing inhouse could be cost-effective in terms of both resources and time.

An understanding of the compositional profile and the interrelated chemical structures of nonoxynol-9 can be gained by considering its industrial manufacture, which begins with the alkylation of phenol with a mixture of isomeric nonenes in the presence of an acid catalyst (Figure 1). The resulting product, a complex mixture of predominately para-substituted nonylphenols, is then subjected to a polymerization reaction with ethylene oxide. This reaction engages the phenol hydroxyl group, resulting in repeating ethylene oxide units of varying length, with the largest fraction of poly(ethylene oxide) repeats being nine (hence the name nonoxynol-9) [14, 15]. The compositional complexity of nonoxynol-9, coupled with its surfactant-like properties and non-volatility have hindered both its detection via GC-MS and its detailed characterization of its mass spectral signature. Determination of the chromatographic profiles and the detailed mass spectral fragmentation patterns of the various components is an important first step towards the development of methods that can be routinely used for the analysis of evidence. Reported herein is a protocol that couples ultrasonic extraction with GC-MS analysis to detect and characterize nonoxynol-9 in a variety of lubricant formulations. Mass spectral characterization of nonoxynol-9 by electron impact ionization enabled a detection limit of $2.14 \mu \mathrm{g}$ for nonoxynol-9.

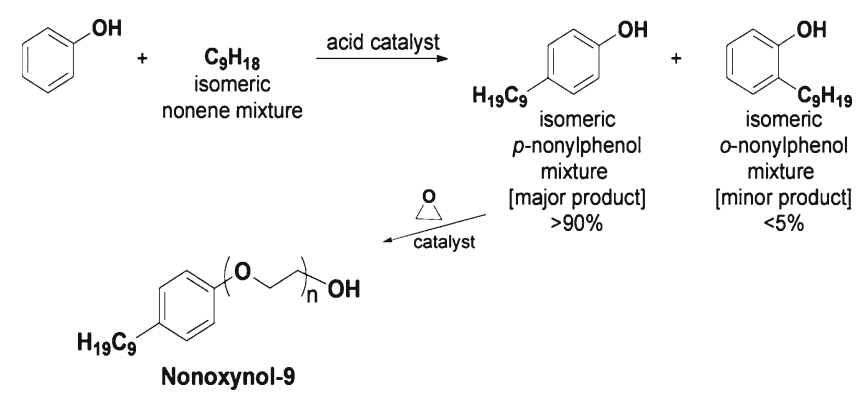

Figure 1. Commercial synthesis of nonoxynol-9. Phenol is reacted with isomeric nonenes, resulting in a mixture of isomeric nonylphenols. The $p$-substituted nonylphenols are then reacted with ethylene oxide, resulting in a complex mixture of congeners (with $n$ varying from 3-17), each of which is comprised of a mixture of nonyl isomers

\section{Experimental}

\author{
GC-MS Analysis
}

Analyses were carried out on a Hewlett Packard (Avondale, PA, USA) 6890 series GC/5972A series mass selective detector. The GC column was a HP-5 $(30 \mathrm{~m} \times 0.25 \mathrm{~mm} \times$ $0.25 \mu \mathrm{m})$ with a $300{ }^{\circ} \mathrm{C}$ injector temperature. Sample aliquots $(2 \mu \mathrm{L})$ were injected with a 1:10 split ratio. Three min post-injection, the oven temperature was ramped from $75^{\circ} \mathrm{C}$ to $300{ }^{\circ} \mathrm{C}$ at a rate of $5{ }^{\circ} \mathrm{C} / \mathrm{min}$ and held at $300{ }^{\circ} \mathrm{C}$ for $60 \mathrm{~min}$. Detection was achieved in electron impact mode and full scan monitoring ( $\mathrm{m} / \mathrm{z} 40-700)$.

\section{Materials}

Nonoxynol-9 was purchased from Spectrum Chemical (New Brunswick, NJ). Condoms and spermicidal jelly were purchased at a local drug store.

\section{Preparation of Nonoxynol-9 Extracts}

Three different samples of nonoxynol-9 were prepared for analysis. Solutions of neat nonoxynol-9 were prepared by sonicating the neat sample $(0.3340 \mathrm{~g})$ in $5.0 \mathrm{~mL}$ of dichloromethane (DCM). DCM extracts of nonoxynol-9 derived from Gynol II jelly were prepared by sonicating $0.5287 \mathrm{~g}$ of the jelly in $5.0 \mathrm{~mL}$ of DCM. The volume was reduced by $100 \times$ by solvent evaporation. The concentrated sample was then subjected to GC-MS analysis. DCM extracts from Trojan Ultra-ribbed condoms were prepared by collecting lubricant with a cotton swab, and suspending the swab in a tube containing $4 \mathrm{~mL}$ of DCM. The resulting sample was sonicated for $20 \mathrm{~min}$ and subjected to GC-MS.

\section{Detection Limits of Nonoxynol-9}

Nonoxynol-9 $(106.81 \mathrm{mg})$ was dissolved in $10.0 \mathrm{~mL}$ of DCM $(10.68 \mathrm{mg} / \mathrm{mL})$. Multiple dilutions were prepared, including a 1:5 dilution of this stock solution $(2.14 \mathrm{mg} / \mathrm{mL})$. Detection limits were calculated based on the weight of nonoxynol-9 injected into the GC-MS (e.g., $1 \mu \mathrm{L}$ of a $2.14 \mathrm{mg} / \mathrm{mL}$ solution is $2.14 \mu \mathrm{g}$ injected).

\section{Results and Discussion}

Trace lubricant evidence associated with sexual assault can originate from a condom or supplementary use of a lubricant. The variety of lubricant formulations that are available is considerable, not only between products from the same company, but also between various brands. Nonoxynol-9 from three sources was solubilized, extracted, and identified. After suspension in dichloromethane, neat nonxynol-9, spermicidal jelly, and lubricant from a condom containing the spermicide, were each sonicated and analyzed by GC-MS (Figure 2). The chromatograms each show a series of regularly spaced clusters of peaks. Each cluster corresponds to a specific length of the poly 

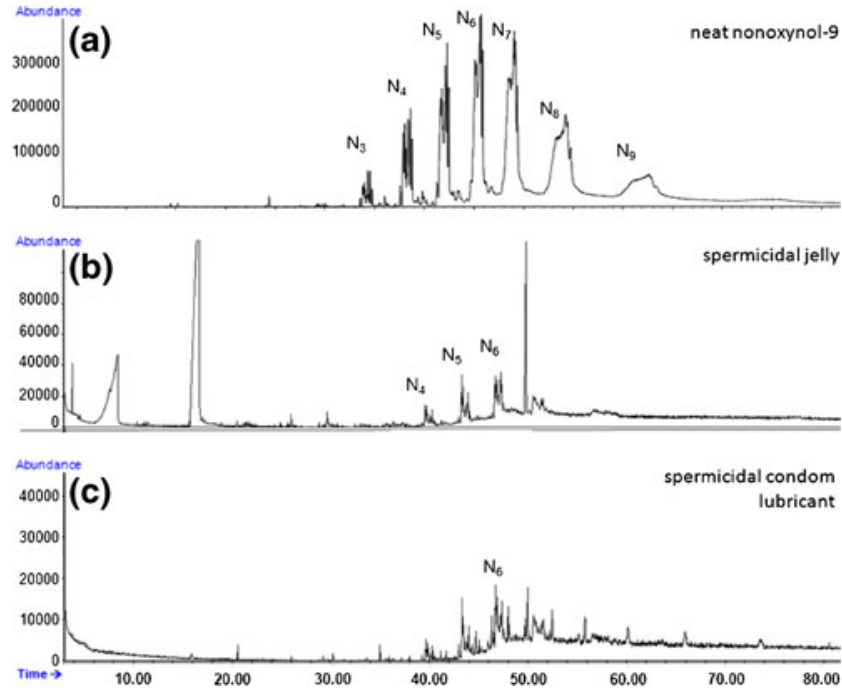

Figure 2. Chromatograms showing components of nonoxynol-9 extracted with dichloromethane, followed by sonication. (a) Nonoxynol-9; (b) spermicidal jelly; (c) spermicidal condom lubricant. The peak clusters represent the indicated poly(ethylene oxide) number $\left(\mathrm{N}_{3}, \mathrm{~N}_{4}, \mathrm{~N}_{5}\right.$, etc.), with each individual peak in the cluster representing a unique $\mathrm{C}_{9} \mathrm{H}_{19}$ isomer

(ethylene oxide) side chain and each peak within a cluster to a specific $\mathrm{C}_{9} \mathrm{H}_{19}$ (nonyl) isomer. In all the samples, components larger than $\mathrm{N}_{9}$ were not observed in the chromatograms due to the decreased volatility associated with the increased length of the ethylene oxide repeat units. The three panels in Supplementary Figure 1 each show the mass spectrum of a single component (a single peak) from the $\mathrm{N}_{3}, \mathrm{~N}_{4}$, and $\mathrm{N}_{5}$ clusters in the chromatogram of neat nonoxynol-9 (Figure 2a), corresponding to the poly(ethylene oxide) repeats of three, four, and five units, respectively. Comparison of the three spectra in Supplementary Figure 1 reveals a number of trends: (1) all the spectra exhibit a weak but identifiable parent peak $\left(\mathrm{M}^{+}\right)$; (2) the individual peaks within each cluster show parent peak increases associated with elongation by a subsequent ethylene oxide repeat, increasing the molecular weight by 44 mass units (e.g., the $\mathrm{M}^{+}$. of $\mathrm{N}_{3}=352$, $\mathrm{N}_{4}=396, \mathrm{~N}_{5}=440$, etc.); (3) the fragmentation pattern due to the $\mathrm{C}_{9} \mathrm{H}_{19}$ side chain is specific to each isomer (detailed later in this section); and (4) fragmentation via cleavage of one, two, or three ethylene oxide $\left(-\mathrm{CH}_{2}-\mathrm{CH}_{2}-\mathrm{O}-\right)$ units, with charge site retention in the ethylene oxide fragments, yields prominent peaks with $m / z$ values of 45,89 , and 133 , respectively.

The fragmentation patterns due to the $\mathrm{C}_{9} \mathrm{H}_{19}$ side chains are also noteworthy. First, the mass spectra in Supplementary Figure 1 reveal information about the preferred fragmentation and charge site retention, showing different hydrocarbon fragments lost within each mass spectrum. The differences in the alkyl fragments lost when comparing one spectrum to another are reflective of differences in branching in the nonyl chains. Second, the three mass spectra in Supplementary Figure 1 exhibit peaks at $\mathrm{m} / \mathrm{z}$ values of $107,121,135$, and 149. These peaks represent the core structure, minus the ethylene oxide side chain, with the respective loss of $\mathrm{C}_{8} \mathrm{H}_{17}$, $\mathrm{C}_{7} \mathrm{H}_{15}, \mathrm{C}_{6} \mathrm{H}_{13}$, and $\mathrm{C}_{5} \mathrm{H}_{11}$ alkyl fragments, with charge site retention on the phenolic fragment. The identities of these peaks were confirmed based on the mass spectra of nonylphenol standards (data not shown) [16].

Ultimately, the structural differences between the nonyl chains of isomeric nonoxynol-9 molecules are best illustrated by comparing the mass spectra of peaks within the same cluster. Accordingly, an expansion of the chromatogram in Figure 2a is shown in Supplementary Figure 2, focusing on the retention time between 37.00 and $40.00 \mathrm{~min}$. The occurrence of nine different peaks in this cluster signifies that nine $\mathrm{C}_{9}$-isomers are resolved in the chromatogram, all of which have the $\mathrm{N}_{4}$ repeat. Each of the spectra shown exhibits a parent peak at $\mathrm{m} / \mathrm{z} 396$, indicating that all three compounds are isomers of $\mathrm{N}_{4}$. However, as would be expected for isomers that differ only in terms of branching within the alkyl chain, different peaks and peak intensities are observed related to the loss of common alkyl fragments and unique hydrocarbon fragmentations. For example, whereas the mass spectra of peaks 4 and 6 (indicated with green and purple arrows, respectively, in Supplemental Figure 2a), show loss of a propyl radical $(\mathrm{m} / \mathrm{z} 353)$, this mass is absent in the spectrum of peak 2 (red arrow). Additionally, all three spectra exhibit charged fragments from loss of $\mathrm{C}_{2} \mathrm{H}_{5}(\mathrm{~m} / \mathrm{z} 367), \mathrm{C}_{5} \mathrm{H}_{11}(\mathrm{~m} / \mathrm{z}$ $325)$, and $\mathrm{C}_{6} \mathrm{H}_{13}(\mathrm{~m} / \mathrm{z} 311)$, but the relative intensities of these fragments are different from one another.

Although nonoxynol-9 has been incorrectly presented as having an unbranched nonyl side chain $[4,13]$, the chromatograms shown herein illustrate its inherent complexity. In total, both of the substituents branching off the core phenol structure of nonoxynol-9 are variable, making the overall "structure" an even more convoluted "mixture of mixtures," best described as the generic chemical structure in Figure 1. Because nonoxynol9 is not a single compound but a complex mixture, a molecular weight cannot be determined, and this complicates any discussion of detection limits in the classical sense. Thus, detection limits were obtained based on sample mass, as well as analysis of both the chromatograms and mass spectra (Supplementary Figure 3). We observed a detection limit of $2.14 \mu \mathrm{g}$. Samples below this mass could not be conclusively identified due to a lack of peaks in the chromatogram and/or fragments that could not be identified within the mass spectral profile. Absolute quantitation of samples was based on serial dilutions of a stock solution (weight/volume), together with a known volume injected. To put this detection limit value into perspective, a conservative measure of the weight of lubricant on a condom is $150 \mathrm{mg}$ [17], such that lubricant containing 7\% nonoxynol-9 is associated with a spermicide content of $\sim 10.5 \mathrm{mg}$ per condom. A study on the amount of residual spermicide remaining within the vagina $4 \mathrm{~h}$ after application showed that at a minimum, a recovery of $\sim 15 \%$ of nonoxynol-9 is possible [18]. Thus, for a condom containing 7\% nonoxynol-9, an amount of $1.6 \mathrm{mg}$ of material would be available for collection with a rape test kit. The detection limit reported herein is $\sim 1000 \times$ lower than this value and is a reasonable amount of material for a sexual 
assault nurse to collect with a swab $[3,6]$. In addition, detection limits in the microgram range are in good agreement with detection levels by GC-MS previously reported for polydimethylsiloxane and polyethylene glycol, which were analyzed as trace evidence from lubricants associated with sexual assault [3].

The ability to perform GC-MS analysis of nonoxynol-9 is complicated by its amphiphilic nature and documented nonvolatility. Because its structure is both hydrophobic and hydrophilic, nonoxynol-9 forms micelles, which complicates its solubility [15]. Additionally, the long hydrocarbon side chains are engaged in intermolecular van der Waals interactions that negatively impact transfer of molecules into the gas phase. Consequently, the most illustrative mass spectral data of nonoxynol- 9 to date have been obtained by LC-MS and desorption chemical ionization MS, which yielded chromatograms in which the individual isomeric components were not resolved [4, 5]. These techniques employ softer ionization methods that produce primarily molecular ions and simpler spectra with little to no fragmentation when compared with EI-based methods [6, 10-13]. The incorporation of a simple sonication step during the extraction was designed to disrupt micelles to better solubilize the material. The chromatographic profiles observed are in agreement with industrial manufacture of nonoxynol-9, which results in a distribution of ethylene oxide repeat units, with the lowest detectable components being those corresponding to $\mathrm{N}_{3}$ (reflecting the presence of three ethylene oxide repeats). Our results confirm a literature report that the industrial synthesis of nonoxynol-9 results in a Poisson distribution of compounds, with only trace levels of $\mathrm{N}_{0}, \mathrm{~N}_{1}$, and $\mathrm{N}_{2}$ being detected in the commercial product [15]. As such, the chromatograms of nonoxynol-9 show clusters of peaks centering on each poly(ethylene oxide) subunit, beginning at $\mathrm{N}_{3}$ (Figure 2). The spermicidal jelly and condom lubricant show a similar chromatographic signature in terms of their distribution and profile, with slight shifts in retention time. The appearance of other peaks in the chromatograms of the spermicidal jelly (at retention times of 8.344 and $16.558 \mathrm{~min}$ ) are consistent with the increased complexity of the matrix compared with that of neat nonoxynol-9. The presence of these peaks in no way precluded the observation of the nonoxynol-9 signature, and can serve as the basis for further studies on individualization of lubricant components from different sources.

The data presented herein provide direct insight into the complexity of the spermicide nonoxynol-9, and for the first time, establish the detailed signature of the substance via GC-MS $[14,15]$. The instrumentation employed is common to crime laboratories where electron impact ionization is routinely used, and the chromatograms and mass spectra of each isomer can be visualized in a manner not possible with softer ionization techniques. Recommendations have been made that sexual assault nurses take multiple swabs from a victim to allow not only for DNA analysis, but also for analysis of other trace evidence such as lubricants [1, 2]. Studies into continued identification of individual isomers of nonoxynol-9 and their limits of detection in the context of individualization of lubricant formulations associated with trace sexual assault evidence are ongoing.

\section{Acknowledgments}

The authors thank the University at Albany, State University of New York, for the funds to support this project, and Professors Daniele Fabris and Li Niu for reviewing the manuscript and providing helpful comments.

\section{References}

1. Blackledge, R.D.: Condom Trace Evidence: A New Factor in Sexual Assault Investigations. FBI Law Enforcement Bulletin 1-6 (1996)

2. Blackledge, R.D.: Collection and Identification Guidelines for Traces from Latex Condoms in Sexual Assault Cases. Crime Laboratory Digest 21, 57-61 (1994)

3. Campbell, G.P., Gordon, A.L.: Analysis of Condom Lubricants for Forensic Casework. J. Forensic Sci. 52, 630-642 (2007)

4. Maynard, P., Allwell, K., Roux, C., Dawson, M., Royds, D.: A protocol for the forensic analysis of condom and personal lubricants found in sexual assault cases. Forensic Sci. Int. 124, 140-156 (2001)

5. Blackledge, R.D., Vincenti, M.: Identification of polydimethylsiloxane lubricant traces from latex condoms in cases of sexual assault. $J$. Forensic Sci. Soc. 34, 245-256 (1994)

6. Shen, Z., Thomas, J.J., Siuzdak, G., Blackledge, R.D.: A Case Study on Forensic Polymer Analysis by DIOS-MS: The Suspect Who Gave Us the Slip. J. Forensic Sci. 49, 1028-1035 (2004)

7. Apostolov, A., Hristov, S., Angelova, E.: DNA Identification of Biological Traces and Interpretation in a Sexual Assault Case. Am. J. Forensic Med. Path. 30, 57-60 (2009)

8. Brauner, P., Gallili, N.: A Condom-The Critical Link in a Rape. $J$. For. Sci. 38, 1233-1236 (1993)

9. Wolfe, J., Exline, D.L.: Characterization of Condom Lubricant Components Using Raman Spectroscopy and Raman Chemical Imaging. J. Forensic. Sci. 48, 1065-1074 (2003)

10. Thomas, J.J., Blackledge, R.D., Siuzdak, G.: Desorption-Ionization on Silicon Mass Spectrometry: An Application in Forensics. Anal. Chem. Acta 442, 183-190 (2001)

11. Bradshaw, R., Wolstenholme, R., Blackledge, R.D., Clench, M.R., Ferguson, L.S., Francese, S.: A novel matrix-assisted laser desorption/ ionisation mass spectrometry imaging based methodology for the identification of sexual assault suspects. Rapid Commun. Mass Spectrom. 25, 415-422 (2011)

12. Hollenbeck, T., Siuzdak, G., Blackledge, R.D.: Electrospray and MALDI Mass Spectrometry in the Identification of Spermicides in Criminal Investigations. J. Forensic. Sci. 44, 783-788 (1999)

13. Spencer, S.E., Kim, S.Y., Kim, S.B., Schug, K.A.: Matrix-assisted laser desorption/ionization-time of flight-mass spectrometry profiling of trace constituents of condom lubricants in the presence of biological fluids. Forensic. Sci. Int. 207, 19-26 (2011)

14. Walter, B.A., Digenis, G.A.: High-Performance Liquid Chromatographic (HPLC) Analysis of Oligomeric Components of the Spermicide Nonoxynol-9. Pharmaceut. Res. 8, 409-411 (1991)

15. Iyer, V., Poddar, S.S.: Update on nonoxynol-9 as vaginal spermicide. Eur. J. Contracept. Reprod. Health Care. 13, 339-350 (2008)

16. Wheeler, T.F., Heim, J.R., LaTorre, M.R., Janes, A.B.: Mass Spectral Characterization of p-Nonylphenol Isomers Using High-Resolution Capillary GC-MS. J. Chromatogr. Sci. 35, 19-30 (1997)

17. Keil, W.: Condom Trace Evidence in Sexual Assaults: Recovery and Characterization. In: Blackledge, R.D. (ed.) Forensic Analysis on the Cutting Edge: New Methods for Trace Evidence Analysis, pp. 81-113. John Wiley and Sons, Inc., Hoboken, NJ (2007)

18. Mauck, C.K., Allen, S., Baker, J.M., Barr, S.P., Abercrombie, T., Archer, D.F.: An evaluation of the amount of nonoxynol-9 remaining in the vagina up to $4 \mathrm{~h}$ after insertion of a vaginal contraceptive film (VCF $\mathbb{R})$ containing 70 mg nonoxynol-9. Contraception 56, 103-110 (1997) 